Received: $\quad 2016.03 .27$

Accepted: 2016.06.13

Published: 2017.02 .04

Authors' Contribution Study Design A Data Collection B Statistical Analysis C Data Interpretation D Manuscript Preparation E Literature Search F Funds Collection G

\title{
MicroRNA-495 Inhibits Gastric Cancer Cell Migration and Invasion Possibly via Targeting High Mobility Group AT-Hook 2 (HMGA2)
}

BCDEF 1 Huashe Wang

BCDF 1 Zhipeng Jiang

BCF 2 Honglei Chen

CF 1 Xiaobin Wu

BF 1 Jun Xiang

ADE 1 Junsheng Peng
1 Department of Gastrointestinal Surgery, The Sixth Affiliated Hospital of Sun Yat-Sen University, Guangzhou, Guangdong, P.R. China

2 Department of Digestive Endoscopic Center, The Sixth Affiliated Hospital of Sun Yat-Sen University, Guangzhou, Guangdong, P.R. China
Corresponding Author: Source of support:

Background: Gastric cancer is one of the most common malignancies, and has a high mortality rate. miR-495 acts as a suppressor in some cancers and HMGA2 (high mobility group AT-hook 2) is a facilitator for cell growth and epithelial-mesenchymal transition (EMT), but little is known about their effect in gastric cancer. This study aimed to investigate the role and mechanism of miR-495 in gastric cancer.

Material/Methods: miR-495 levels were quantitatively analyzed in gastric cancer tissue and GES-1, SGC-7901, BGC-823, and HGC27 cell lines by qRT-PCR. Levels of miR-495 and HMGA2 were altered by cell transfection, after which cell migration and invasion were examined by Transwell and E-cadherin (CDH1); vimentin (VIM), and alpha smooth muscle actin (ACTA2) were detected by qRT-PCR and Western blotting. The interaction between miR-495 and HMGA2 was verified by dual-luciferase reporter assay.

Results: $\quad$ miR-495 was significantly downregulated in cancer tissue and cell lines $(p<0.05)$. Its overexpression inhibited cell migration and invasion, elevated CDH1, and inhibited VIM and ACTA2 levels in BGC-823 and HGC-27 cells. miR-495 directly inhibited HMGA2, which was upregulated in gastric cancer tissue, and promoted cell migration and invasion, inhibited CDH1, and elevated VIM and ACTA2.

Conclusions: miR-495 acts as a tumor suppressor in gastric cancer by inhibiting cell migration and invasion, which may be associated with its direct inhibition on HMGA2. These results suggest a promising therapeutic strategy for gastric cancer treatment.

MeSH Keywords: $\quad$ Epithelial-Mesenchymal Transition • HMGA2 Protein • MicroRNAs • Neoplasm Invasiveness • Stomach Neoplasms • Transcellular Cell Migration

Full-text PDF: http://www.medscimonit.com/abstract/index/idArt/898740

官当 3002 豊尚




\section{Background}

Gastric cancer is one of the most common malignancies worldwide and the second most important cause of tumor death after lung cancer, with an average five-year survival rate of less than 20\% [1]. The highest incidence rate is found in East Asian countries such as China, Japan, and Korea. Risk factors include Helicobacter pylori infection (which is a predominant risk factor), dietary factors, tobacco use, and obesity [2]. Few specific symptoms can be observed in the earlier stages of gastric cancer, which can often lead to a delayed diagnosis. In addition, genetic complexity and heterogeneity of gastric cancer adds to the difficulty of effective treatment. Recent studies have uncovered blood-related factors $[3,4]$ and possible cellular targets for drug treatment, such as vascular endothelial growth factor and epidermal growth factor receptor [5], but the biology and treatment options of gastric cancer still need further study.

Recent studies found evidence that the progression and development of gastric cancer is closely related to the process of epithelial-mesenchymal transition (EMT) $[6,7]$, a process also involved in other diseases [8,9]. Activated EMT endows gastric cancer cells with increased mesenchymal characteristics and promotes cell invasion and metastasis [6]. During EMT, the $\mathrm{CDH} 1$ gene-encoded $\mathrm{E}$-cadherin switches to $\mathrm{N}$-cadherin, which is usually expressed in mesenchymal cells [10]. Vimentin (VIM) is a positive regulator of EMT and a potential prerequisite for EMT induction [11]. Alpha smooth muscle actin (ACTA2) is also an early sign of EMT. A previous study indicated that HMGA2 (the high mobility group AT-hook 2) was a promotive factor in many malignant neoplasms, and induced EMT in gastric cancer [12]. However, additional research is required to reveal the complicated mechanisms involved in gastric cancer.

MicroRNAs (miRNAs) are a group of small non-coding RNAs that exhibit powerful abilities in gene regulation via repressing translation or degrading mRNAs. Due to their effective regulation of gene expression, miRNAs play vital roles in modulating various diseases, including gastric cancer, through affecting cell cycle, proliferation, migration, invasion, and apoptosis, and further changing tumorigenesis and cancer metastasis [13]. For example, let-7 miRNA modulates an important RNA-binding protein Lin28 and influences the biological behavior of gastric cancer [14]. miR-495 plays a suppressive role in the post-transcription of FOXC1 (forkhead box C1) and thus inhibits endometrial cancer progression [15]. miR-495 has been shown in recent studies to participate in regulating gastric cancer cells via direct interaction with PRL-3 (phosphatase of regenerating liver-3) $[16,17]$. So miR-495 appears to be active in gastric cancer cells, although the specific mechanisms are as yet unknown.
The aim of this study was to analyze the roles and pivotal mechanisms of miR-495 in gastric cancer. The expression pattern of miR-495 was examined in gastric cancer tissue and gastric cell lines of different differentiation degrees. Cell migration and invasion were detected after altering miR-495 levels in BGC823 and HGC-27 cells. The aforementioned HMGA2 was predicted to be a target for miR-495 and we confirmed this interaction by luciferase reporter assay. The study results suggest a potential therapeutic strategy for gastric cancer treatment and add to the knowledge of the role of miRNAs in modulating gastric cancer.

\section{Material and Methods}

\section{Tissue samples}

Human gastric cancer tissue and normal gastric tissue were collected from Tianjin Medical University Cancer Institute \& Hospital (Tianjin, China). A total of 40 gastric cancer tissue samples from gastric cancer patients and 10 normal tissue samples from patients without gastric cancer were collected during gastrointestinal surgery or gastroscopy with the patients' informed consent. The patients did not undergo radiotherapy or chemotherapy before their surgery. The 40 gastric cancer tissue samples included high-, moderate-, low-differentiation and un-differentiated tissue, each group containing 10 samples. The gastric cancer stages of the tissue samples were assessed by double-blind protocol by two pathologists according to the TNM staging system proposed by the Union for International Cancer Control/American Joint Committee on Cancer (UICC/AJCC) [18]. No apparent differences existed in the gender, age, or TNM stage between any two groups. This study was approved by a local ethics committee and performed based on the instructions of our institute.

\section{Cells}

Human gastric mucosal cell line GES-1 (Saierbio, Tianjing, China) and human gastric cancer cell lines SGC-7901, BGC-823, and HGC-27 (ATCC, Manassas, VA, USA) were used in this study for in vitro analyses. SGC-7901 had moderate differentiation, BGC-823 had low differentiation, and HGC-27 was undifferentiated [19]. The three cell lines were examined for levels of miR-495 expression. The cell lines were all cultured in Roswell Park Memorial Institute (RPMI)-1640 medium supplemented with $10 \%$ fetal bovine serum (FBS), $2 \mathrm{mM}$ glutamine, $100 \mathrm{U} / \mathrm{mL}$ penicillin, and $100 \mathrm{mg} / \mathrm{mL}$ streptomycin (Gibco, Carlsbad, CA, USA) in humidified atmosphere with $5 \% \mathrm{CO}_{2}$ at $37^{\circ} \mathrm{C}$. The cells were passaged at a confluency of $70 \%$. 


\section{Cell transfection}

Cell transfection was performed to alter the level of miR-495 or HMGA2 in cell lines BGC-823 and HGC-27. The miR-495-specific precursor miRNA (pre-miR495), antisense oligo (ASO) and the corresponding controls were synthesized by Ambion (Carlsbad, CA, USA) to alter the miR-495 levels. The HMGA2specific small interference RNA (siRNA) was synthesized by GenePharma (Shanghai, China) to inhibit the HMGA2 level. The coding sequence of HMGA2 was cloned into pcDNA3.1 vector (Thermo Scientific, Carlsbad, CA, USA) and sequenced to verify the correct ligation, and then pcDNA3.1-HMGA2 was transfected to overexpress HMGA2 and the blank vector was used as a control. Transfection was performed in antibioticfree medium in 96-well plates with the help of Lipofectamine 2000 (Invitrogen, Carlsbad, CA, USA) according to the manufacturer's instructions. Each transfection system contained $5 \times 10^{4}$ cells, $2 \mu \mathrm{L}$ Lipofactamine 2000 and pre-miR-495 (10 nM), siHMGA2 (20 nM), or pcDNA3.1-HMGA2 (200 ng). Further analyses were performed at 48 hours after transfection.

\section{Luciferase reporter assay}

The interaction between HMGA2 3'UTR and miR-495 was predicted by TargetScanHuman 7.0 (www.targetscan.org). To test the binding site of HMGA2 and miR-495, five bases in HMGA2 3'UTR were mutated using QuickChange Multi Site-Directed Mutagenesis Kit (Agilent Technologies, Santa Clara, CA, USA), and dual-luciferase reporter assay was performed on the wildtype (wt) or mutant (mt) HMGA2 3'UTR in BGC-823 and HGC27 cells using the kit Dual-Luciferase Reporter Assay (Promega, Madison, WI, USA) according to the manufacturer's instructions. The intensity of fluorescence signals was detected by a GENios Pro microplate reader (Tecan, Männedorf, Switzerland).

\section{Transwell assay}

Transwell assay was performed to detect the migration and invasion abilities of transfected BGC-823 and HGC-27 cells using Transwell sets ( $8 \mu \mathrm{m}, \mathrm{BD}$ Biosciences, San Jose, CA, USA). For cell invasion assay, Matrigel (BD Biosciences) was pre-coated to the upper side of the membrane, incubated at $37^{\circ} \mathrm{C}$ for 30 minutes for gel formation, and hydrated in serum-free medium for one hour before use. The cells were digested, washed in phosphate-buffered saline (PBS), resuspended in serumfree medium, and seeded in the upper chamber at a density of $5 \times 10^{5}$ cells $/ \mathrm{mL}$. The lower chamber was filled with medium containing $20 \% \mathrm{FBS}$, and then the set was assembled and incubated at $37^{\circ} \mathrm{C}$ for 24 hours. After incubation, the membrane was washed in PBS, fixed in methanol for 30 minutes, and dried. The cells remaining on the upper side were wiped away with cotton swabs, and the cells that had crossed the membrane were stained in $0.1 \%$ crystal violet (Beyotime, Shanghai,
China) for 20 minutes. Stained cells were observed and counted under an optical microscope (Olympus, Tokyo, Japan). For cell migration assay, the same procedures were conducted but without Matrigel on the membrane.

\section{qRT-PCR}

qRT-PCR was performed to detect the relative levels of has-miR495-3p, HGMA2, CDH1, VIM, and ACTA2 in gastric tissue and cell lines quantitatively. The tissue or cell samples were lysed in TRIzol (Invitrogen, Carlsbad, CA, USA) for total RNA extraction or RNAiso for Small RNA (Takara, Dalian, China) for miRNA extraction. First-strand complementary DNA (cDNA) was synthesized with random primers or the specific reverse transcription primer for hsa-miR-495-3p (5'-CTC AAC TGG TGT CGT GGA GTC GGC AAT TCA GTT GAG AAG AAG TG-3') under the catalysis of PrimeScript Reverse Transcriptase (Takara). qRT-PCR was conducted on a QuantStudio 6 Flex Realtime PCR system (Applied Biosystems, Carlsbad, CA, USA), with 20 ng of cDNA and the specific primers for miR-495-3p (Fw: 5'-ACA CTC CAG CTG GGA AAC AAA CAT GGT GCA-3' and Rv: 5'-TGG TGT CGT GGA GTC G-3'), HGMA2 (Fw: 5'-TGG TGC AAG ACT CAG GAG-3' and Rv: 5'-CAG TCG GAA AGC AAA GG-3'), CDH1 (Fw: 5'-GCA TTG CCA CAT ACA CTC TCT TCT-3' and Rv: 5'-TCG GTT ACC GTG ATC AAA ATC TC-3'), VIM (Fw: 5'-ACA TTG AGA TTG CCA CCT AC-3' and Rv: 5'-AAC CGT CTT AAT CAG AAG TGT C-3') or ACTA2 (Fw: 5'-AGC GTG GCT ATT CCT TCG T-3' and Rv: 5'-CTC ATT TTC AAA GTC CAG AGC TAC A-3') in each system. U6 (Fw: 5'-CTC GCT TCG GCA GCA CA-3' and Rv: 5'-AAC GCT TCA CGA ATT TGC GT3') and GAPDH (Fw: 5'-GAA GGT GAA GGT CGG AGT C-3' and Rv: 5'-GAA GAT GGT GAT GGG ATT TG-3') were used as the internal references for miR-495 and other genes, respectively. Data were analyzed with the $2^{-\Delta \Delta c t}$ method.

\section{Western blot}

Total protein from tissue samples and cells were extracted by M-PER Mammalian Protein Extraction Reagent (Thermo Scientific) according to the manufacturer's instructions. Protein samples $(20 \mu \mathrm{g})$ were separated by sodium dodecyl sulfate-polyacrylamide gel electrophoresis and transferred to polyvinylidene fluoride membranes. The membranes were blocked in 5\% skim milk at room temperature for two hours and then incubated in the specific primary antibodies for HMGA2 (ab97276, Abcam, Cambridge, UK), CDH1 (ab181860), VIM (ab92547), and ACTA2 (ab32575) at $4^{\circ} \mathrm{C}$ overnight. GAPDH (ab8245) was used as an internal reference. After washing in PBS, the membranes were incubated in horseradish peroxidase-conjugated secondary antibody at room temperature for one hour, after which positive signals were developed by EasyBlot ECL Kit (Sangon Biotech, Shanghai, China) and the relative density of bands was analyzed by ChemiDoc XRS System (Bio-Rad, Hercules, CA, USA). 

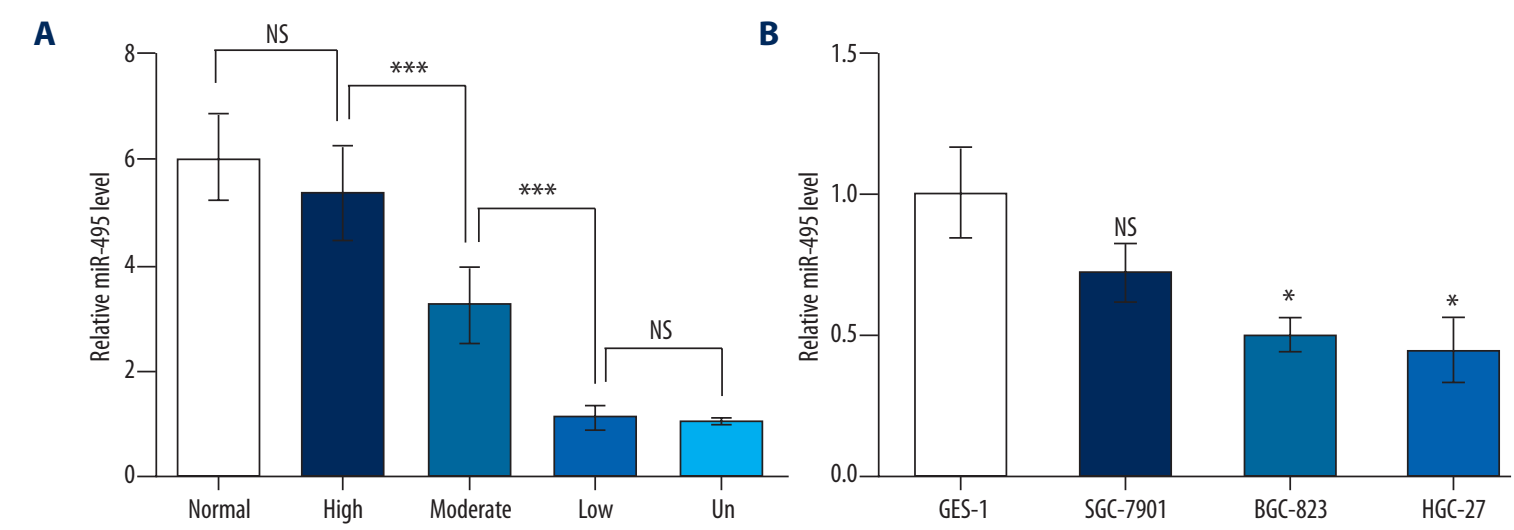

Figure 1. miR-495 was downregulated in gastric cancer tissue and cell lines. (A) miR-495 was significantly downregulated in gastric cancer tissue compared to normal tissue $(n=10)$. The gastric cancer tissue samples were divided into different groups based on their differentiation degree: highly differentiated (high), moderately differentiated (moderate), lowly differentiated (low) or undifferentiated (un). NS, not significant. ${ }^{* *} p<0.001$. (B) miR-495 was significantly downregulated in gastric cancer cell lines BGC-823 and HGC-27. NS - not significant compared to GES-1. ${ }^{*} p<0.05$ compared to GES-1.

\section{Statistical analysis}

All experiments were repeated in triplicate and data were represented as the mean \pm standard deviation. Data were first analyzed by $F$ test for homoscedasticity and then $t$ test for significant difference using SPSS 20 (IBM, New York, USA). $\chi^{2}$ test was performed to examine the differences in gender, age, and TNM stages between any two tissue groups. A value of $p<0.05$ was considered significantly different between groups.

\section{Results}

\section{miR-495 is downregulated in gastric cancer tissue and cell lines}

The expression level of miR-495 was compared in gastric cancer tissue and normal tissue by qRT-PCR and inhibited miR-495 levels were found in cancer tissue of different differentiation degrees (Figure 1A). Although no significant suppression was detected in the highly differentiated tissue compared to normal tissue, miR-495 was markedly downregulated in tissue samples of moderate differentiation compared to high differentiation $(p<0.001)$, and was further inhibited in the low-differentiation and undifferentiated tissue samples $(p<0.001)$. Its level was also examined in normal gastric cell line GES-1 and three gastric cancer cell lines of different differentiation degrees, and a suppressed miR-495 pattern was also observed. No significant downregulation was detected in moderatelydifferentiated SGC-7901, but miR-495 was obviously inhibited in low-differentiated BGC-823 and undifferentiated HGC-27 compared to GES-1 ( $p<0.05$, Figure $1 \mathrm{~B})$. Taken together, there might be a potential correlation between miR-495 level and gastric cancer differentiation degrees. These results suggest that miR-495 might play a role in gastric cancer progression, which was analyzed in additional experiments.

\section{miR-495 inhibits gastric cancer cell migration and invasion}

Because cell lines BGC-823 and HGC-27 possessed marked miR-495 changes, the two cell lines were used in cell transfection to alter miR-495 levels, and the results showed effective upregulation or downregulation of miR-495 by transfecting pre-miR-495 or miR-495 ASO ( $p<0.05$ and $p<0.01$, respectively, Figure 2A). Transwell assays showed that miR-495 overexpression decreased migrated and invasive cell numbers in the two cell lines ( $p<0.05$ and $p<0.01$, respectively, Figure $2 \mathrm{~B}, 2 \mathrm{C}$ ), and its downregulation increased migrated and invasive cells $(p<0.05)$, indicating that miR-495 might suppress cell migration and invasion in gastric cancer cell lines.

Then we examined three EMT-related factors. qRT-PCR showed that the EMT inhibitor $C D H 1$ mRNA was promoted, while the EMT indicators VIM and ACTA2 mRNA levels were inhibited by miR-495 overexpression in BGC-823 cells ( $p<0.01$ and $p<0.05$, respectively, Figure $2 \mathrm{D}$ ). Opposite effects were found when miR495 was inhibited ( $p<0.05$ and $p<0.001$, respectively). Western blot analysis showed similar changing patterns in the protein levels of these factors (Figure 2E). So miR-495 might regulate CDH1, VIM, and ACTA2, and suppress EMT.

\section{HMGA2 is a direct target of miR-495 and has opposite functions against miR-495}

As predicted by TargetScanHuman 7.0, HMGA2 mRNA was one target of miR-495, with the sequence "GUUUGUUG" being the 


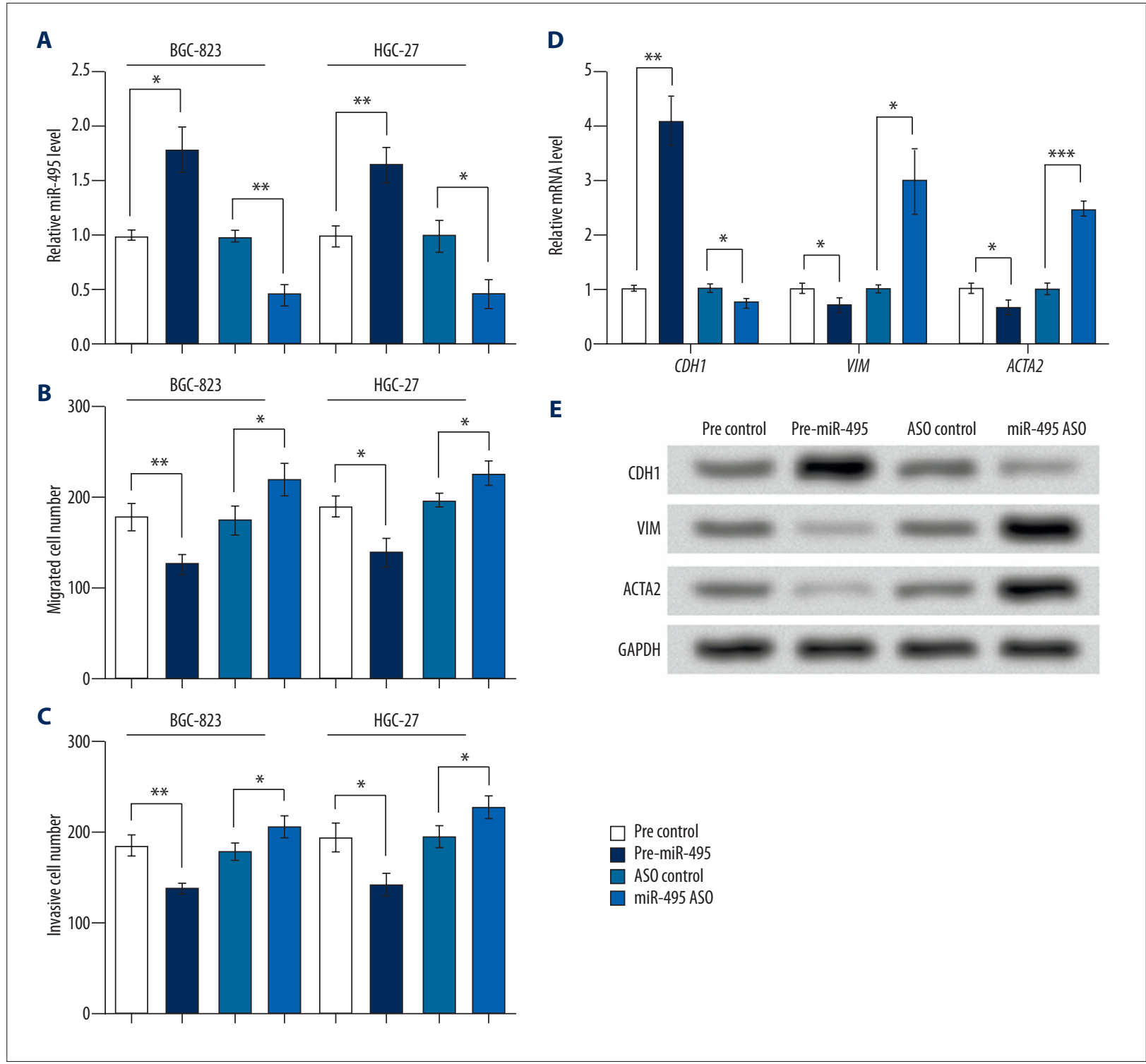

Figure 2. miR-495 inhibited cell migration and invasion in BGC-823 and HGC-27 cell lines and regulated EMT factors. Pre-miR-495, cells were transfected with miR-495 precursor to overexpress miR-495. miR-495 ASO, cells transfected with miR-495 antisense oligo (ASO) to inhibit miR-495. Pre-ASO control, the corresponding control group of pre-miR-495 or miR-495 ASO. qRT-PCR, Western blot and Transwell assays were performed at 48 hours after transfection. (A) miR-495 was successfully promoted or inhibited by cell transfection in BGC-823 and HGC-27 cells. (B) miR-495 inhibited, and its ASO promoted, cell migration in BGC-823 and HGC-27 cells. (C) miR-495 inhibited, and its ASO promoted, cell invasion in BGC-823 and HGC-27 cells. (D) miR495 upregulated $C D H 1$ and downregulated VIM and ACTA2 mRNA levels in BGC-823 cells. (E) miR-495 upregulated CDH1 and downregulated VIM and ACTA2 protein levels in BGC-823 cells. GAPDH mRNA and protein were used as internal references for qRT-PCR and Western blot, respectively. ${ }^{*} p<0.05 .{ }^{* *} p<0.01$. ${ }^{* * *} p<0.001$. CDH1 - E-cadherin. VIM - vimentin. ACTA2 - alpha smooth muscle actin.

predicted binding site. Five bases were mutated (Figure $3 \mathrm{~A}$ ) and both the wildtype and mutant type of HMGA2 3'UTR were applied to dual-luciferase reporter assay. Results showed that in cell lines BGC-823 and HGC-27, the overexpressed miR-495 could only inhibit the luciferase activity of HMGA2 3'UTR-wt $(p<0.01$ and $p<0.05$, respectively, Figure $3 \mathrm{~B}$ ), suggesting that HMGA2 3'UTR-wt, rather than the mutant type, could directly interact with miR-495. Then both mRNA and protein levels of HMGA2 were detected in the transfected BGC-823 cells, and results showed that HMGA2 mRNA and protein expression was inhibited by the upregulated miR-495 and promoted by miR-495 ASO ( $p<0.01$ and $p<0.05$, respectively, Figure $3 C-3 \mathrm{E}$ ). Taken together, this indicates that miR-495 could directly bind to and inhibit HMGA2. 


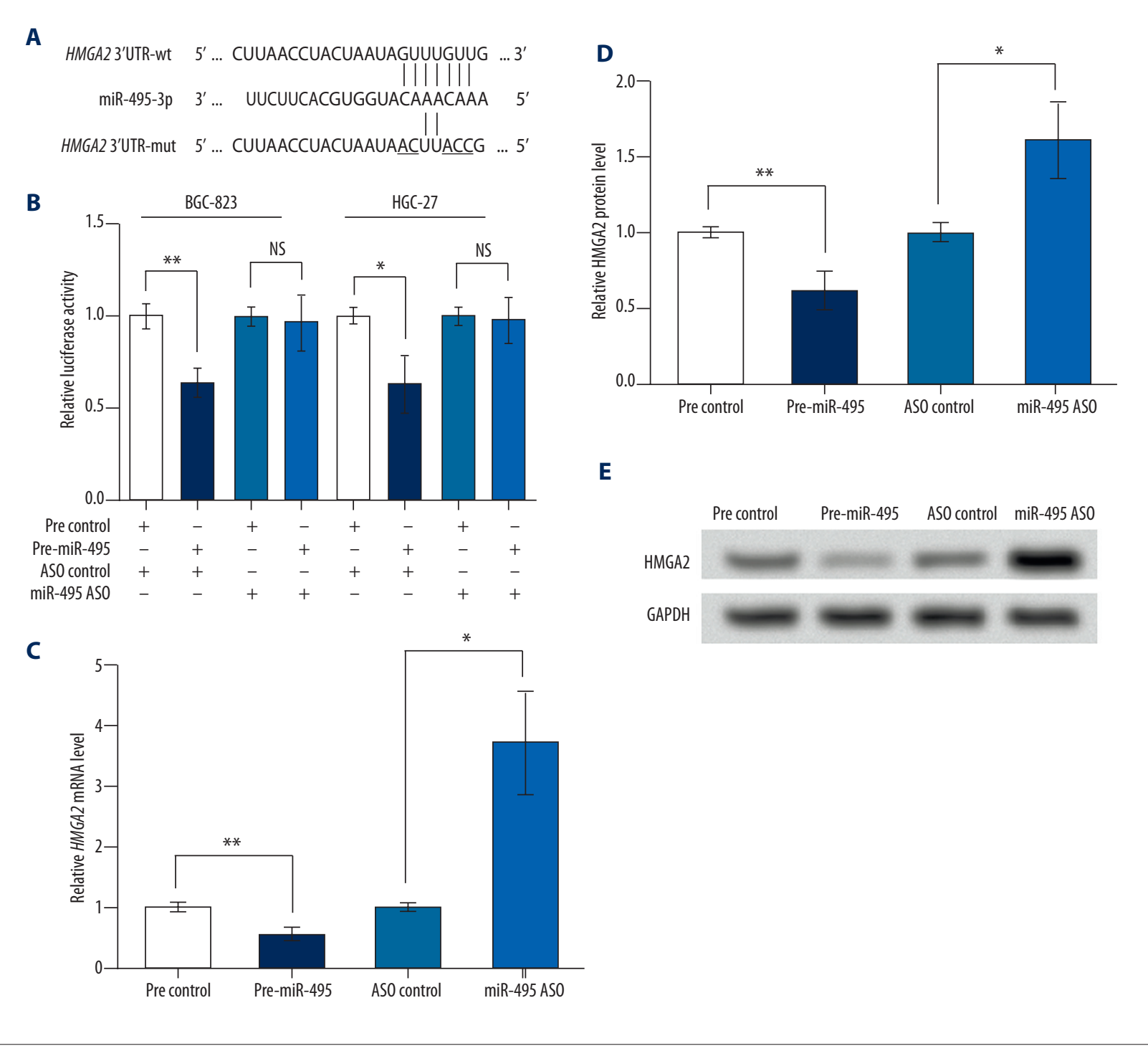

Figure 3. miR-495 directly binds to and inhibits HMGA2. pre-miR-495, cells were transfected with miR-495 precursor to overexpress miR-495. miR-495 ASO, cells transfected with miR-495 antisense oligo (ASO) to inhibit miR-495. Pre-ASO control, the corresponding control group of pre-miR-495 or miR-495 ASO. wt, wildtype. mut, mutant type. (A) A schematic diagram of HMGA2 3'UTR-wt, and HMGA2 3'UTR-mut and their predicted interaction with miR-495. The mutated bases are underlined. (B) Dual-luciferase reporter assay results of altered HMGA2 3'UTR activity by miR-495 in cell lines BGC-823 and HGC27. (C) qRT-PCR showing relative level of HMGA2 mRNA in transfected BGC-823 cells. (D) Relative HMGA2 protein level in transfected BGC-823 cells based on the Western blot result, as shown in (E). GAPDH mRNA and protein were used as internal references for qRT-PCR and Western blot, respectively. NS - not significant. ${ }^{*} p<0.05 .{ }^{* \star} p<0.01$. HMGA2 - high mobility group AT-hook 2.

In gastric cancer tissue, HMGA2 mRNA and protein levels were significantly upregulated compared to the adjacent normal tissue (Figure 4A, 4B). In addition, miR-495 inhibited HMGA2 in gastric cancer cells as mentioned earlier; we suspected that HMGA2 had the opposite effects from miR-495 on BGC-823 and HGC-27 cells, and thus altered HMGA2 expression in the two cell lines (Figure 4C). Actually, HMGA2 overexpression significantly induced the migration and invasion abilities in both cell lines, and si-HMGA2 suppressed these abilities (Figure 4D, 4E).
Besides, HMGA2 inhibited CDH1 and promoted VIM and ACTA2, as shown in qRT-PCR and Western blot results (Figure 4F, 4G). Thus miR-495 and HMGA2 had distinct effects on gastric cancer cells, and HMGA2 might be related to the mechanism of miR-495 in gastric cancer cells. 


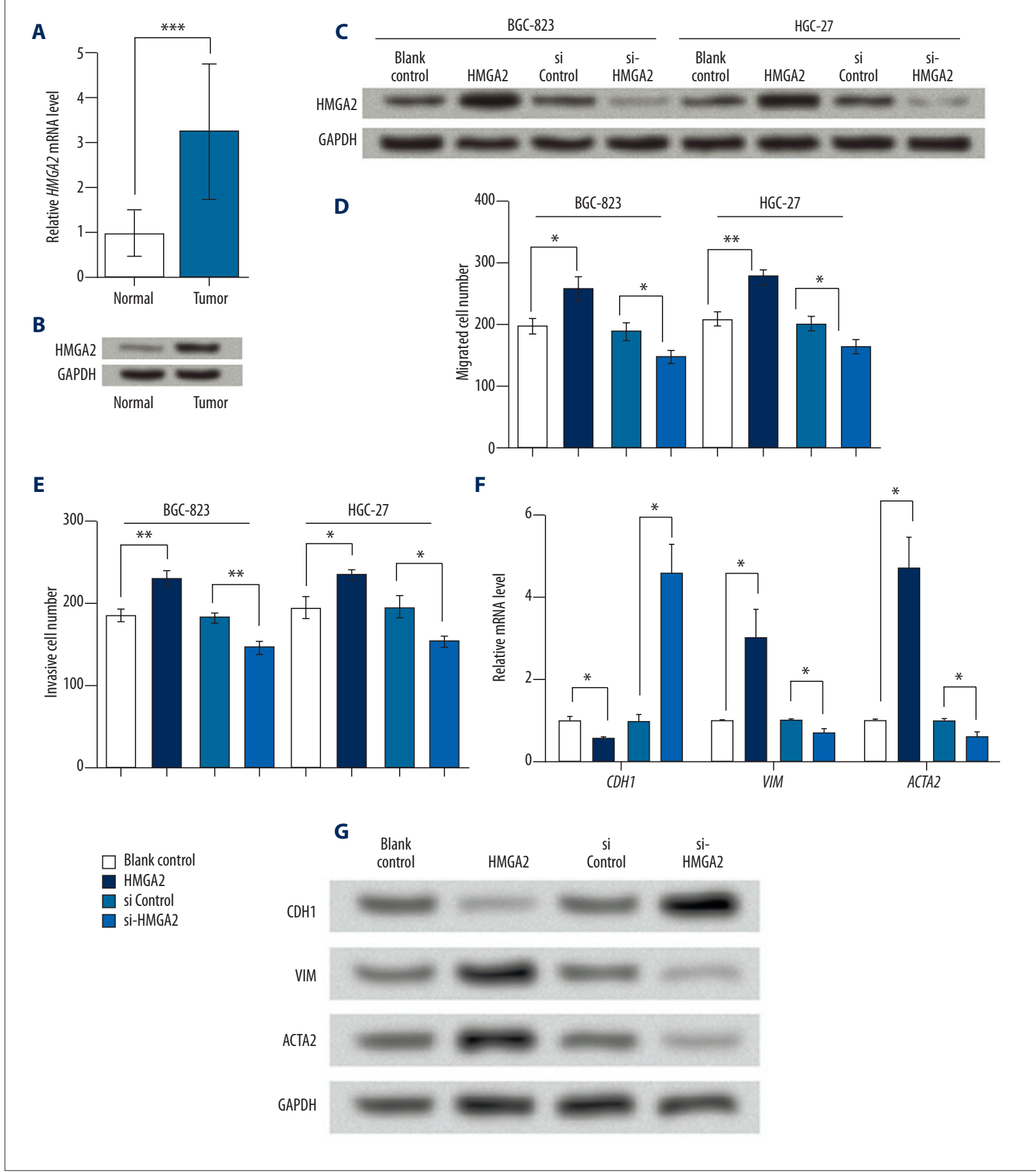

Figure 4. HMGA2 is upregulated in gastric cancer tissue and promotes cell migration and invasion in gastric cancer cell lines. (A) qRTPCR showing relative HMGA2 mRNA level in gastric cancer tissue and the adjacent normal tissue. (B) Western blot showing HMGA2 protein level in gastric cancer tissue and the adjacent normal tissue. (C) HMGA2 protein level was successfully elevated by HMGA2 overexpression vector (HMGA2) and inhibited by its specific siRNA (si-HMGA2). (D) Transwell assay indicating that HMGA2 increased migrated cells in BGC-823 and HGC-27. (E) Transwell assay indicating that HMGA2 increased invasive cells in BGC-823 and HGC-27. (F) HMGA2 promoted CDH1 and inhibited VIM and ACTA2 mRNA levels in BGC-823 cells. (G) HMGA2 promoted CDH1 and inhibited VIM and ACTA2 protein levels in BGC-823 cells. GAPDH mRNA and protein were used as internal references for qRT-PCR and Western blot, respectively. ${ }^{*} p<0.05 .{ }^{* *} p<0.01 .{ }^{* * *} p<0.001 . \mathrm{HMGA}$, high mobility group AT-hook 2. CDH1 - E-cadherin. VIM - vimentin. ACTA2 - alpha smooth muscle actin. 


\section{Discussion}

In the present study, we examined the significantly downregulated level of miR-495 in gastric cancer tissue and cell lines compared to normal tissue and cell lines. The overexpression of miR-495 in cell lines BGC-823 and HGC-27 suppressed cell migration and invasion, and regulated $\mathrm{CDH} 1$, VIM, and ACTA2. HMGA2 was a direct target for miR-495 and its overexpression promoted cell migration and invasion in gastric cancer cell lines, which were effects opposite to miR-495.

As aforementioned, EMT plays a vital role in the progression and development of gastric cancer, and pivotal factors like CDH1 [20,21], VIM [22], and ACTA2 [23] are involved in this process. In our study, miR-495 overexpression led to the elevation of $\mathrm{CDH} 1$ and inhibition of VIM and ACTA2, which might imply a regulated EMT process in the transfected cell lines. A previous study showed that miR-495 inhibits EMT-related factors like Snail and SLUG to suppress EMT [24]. Thus, it is reasonable to speculate that miR-495 may impact EMT in gastric cancer cells, which will be an interesting topic for further investigation.

HMGA2 is a nuclear-binding protein that exhibits vital functions in cell growth and differentiation, and its overexpression has been reported in various neoplasms $[25,26]$. Its regulation on VIM and $\mathrm{CDH} 1$ is involved in overexpression-induced EMT [27]. In accordance with these previous findings, the overexpression of HMGA2 in gastric cancer cells led to expression changes in $\mathrm{CDH} 1, \mathrm{VIM}$, and ACTA2. In addition to the regulation effect of these factors, HMGA2 was upregulated in gastric cancer tissue compared to normal tissue, and its overexpression promoted cell migration and invasion in BGC-823 and HGC-27 cells, suggesting it has a distinct function related to miR-495. Luciferase reporter assay indicated HMGA2 was a direct target for miR495, which further supports that HMGA2 may be involved in the functional mechanism of miR-495 in gastric cancer cells.

In the transfected cells, miR-495 overexpression could inhibit both mRNA and protein levels of HMGA2. Similar results were found in other studies regarding miR-495 regulation of the translation process as well as mRNA stability of its targets $[28,29]$. However, miR-495 only regulates the translation process of some targets, without affecting their mRNA

\section{References:}

1. Correa P: Gastric cancer: Overview. Gastroenterol Clin North Am, 2013; 42: 211-17

2. Crew KD, Neugut Al: Epidemiology of gastric cancer. World J Gastroenterol, 2006; 12: 354-62

3. Lv J, Lv CQ, Xu L, Yang H: Plasma content variation and correlation of plasmalogen and GIS, TC, and TPL in gastric carcinoma patients: A comparative study. Med Sci Monit Basic Res, 2015; 21: 157-60 levels [30]. Studies have found evidence that the sequence signatures, such as A/U content, of target mRNAs are crucial for mRNA degradation induced by miRNAs [31,32]. So based on our results, it is likely that miR-495 inhibits HMGA2 expression via inducing HMGA2 mRNA degradation, or at the same time suppressing the translation progress.

miR-495 was been shown to be downregulated in gastric cancer tissue and cell lines, such as BGC-823 and HGC-27, and it suppressed cell migration and invasion in these two cell lines, suggesting its suppressive role in gastric cancer. Similar effects of miR-495 have been found in SGC-7901 cells, where transfection of miR-495 mimics suppressed cell migration and invasion, as revealed by Transwell assay [16]. In addition, earlier studies detected significant downregulated miR-495 in SGC-7901 cells, while our study also suggested that miR-495 was inhibited in SGC-7901 cells and GES-1 cells, although no significant difference was found. We suspect that the disparity may have been generated from the different methods of miR-495 overexpression, which needs further testing. Similar results have also been reported in leukemia [33], non-small cell lung cancer [34], prostate cancer [35], Dicer-deficient acini, and pancreatitis-induced metaplasia [36], where miR-495 functions as a tumor suppressor inhibiting cell migration and invasion. The control of miR-495 level may rely on the methylation status of the miR-495 gene promoter or the regulation by transcription factors like E12/E47, as found in gastric cancer cells and breast cancer stem cells $[17,37]$. Further research on the functional mechanism and regulation strategies of miR-495 is necessary before applying them to gastric cancer treatment.

\section{Conclusions}

In summary, miR-495 is a tumor suppressor in gastric cancer, suppressing gastric cancer cell migration and invasion, possibly via its direct regulation of HMGA2. This study provides a potential therapeutic strategy focusing on miR-495 for gastric cancer treatment. Future investigations will concentrate on more detailed mechanisms.

\section{Conflicts of interests}

There are no conflicts of interest.

5. Lordick F, Allum W, Carneiro F et al: Unmet needs and challenges in gastric cancer: The way forward. Cancer Treat Rev, 2014; 40: 692-700

6. Peng Z, Wang CX, Fang EH et al: Role of epithelial-mesenchymal transition in gastric cancer initiation and progression. World J Gastroenterol, 2014; 20: $5403-10$ 
7. Zhao L, Li W, Zang W et al. JMJD2B promotes epithelial-mesenchymal transition by cooperating with beta-catenin and enhances gastric cancer metastasis. Clin Cancer Res, 2013; 19: 6419-29

8. Montemayor-Garcia C, Hardin H, Guo Z et al: The role of epithelial mesenchymal transition markers in thyroid carcinoma progression. Endocr Pathol, 2013; 24: 206-12

9. Liang Q, Li L, Zhang J et al: CDK5 is essential for TGF-beta1-induced epithelial-mesenchymal transition and breast cancer progression. Sci Rep, 2013; 3: 2932

10. Rogers CD, Saxena A, Bronner ME: Sip1 mediates an E-cadherin-to- $\mathrm{N}$-cadherin switch during cranial neural crest EMT. J Cell Biol, 2013; 203: 835-47

11. Ivaska J: Vimentin: Central hub in EMt induction? Small GTPases, 2011; 2: 51-53

12. Zha L, Zhang J, Tang W et al: HMGA2 elicits EMT by activating the Wnt/beta-catenin pathway in gastric cancer. Dig Dis Sci, 2013; 58: 724-33

13. Pan H-W, Li S-C, Tsai K-W: MicroRNA dysregulation in gastric cancer. Curr Pharm Des, 2013; 19: 1273-84

14. Song $\mathrm{H}, \mathrm{Xu} \mathrm{W}$, Song J et al: Overexpression of Lin28 inhibits the proliferation, migration and cell cycle progression and induces apoptosis of BGC 823 gastric cancer cells. Oncol Rep, 2015; 33: 997-1003

15. Xu YY, Tian J, Hao Q, Yin LR: MicroRNA-495 downregulates FOXC1 expression to suppress cell growth and migration in endometrial cancer. Tumour Biol, 2016; 37(1): 239-51

16. Li Z, Cao Y, Jie $Z$ et al: miR-495 and miR-551a inhibit the migration and invasion of human gastric cancer cells by directly interacting with PRL-3. Cancer Lett, 2012; 323: 41-47

17. Li Z, Zhang G, Li D et al: Methylation-associated silencing of miR-495 inhibit the migration and invasion of human gastric cancer cells by directly targeting PRL-3. Biochem Biophys Res Commun, 2015; 456: 344-50

18. Edge SB, Byrd DR, Compton CC et al: AJCC Cancer Staging Manual. New York, Springer-Verlag, 2010

19. Yu M, Gou WF, Zhao $S$ et al: Beclin 1 expression is an independent prognostic factor for gastric carcinomas. Tumour Biol, 2013; 34: 1071-83

20. $\mathrm{Yu} \mathrm{H}$, Shen $\mathrm{Y}$, Hong J et al: The contribution of TGF- $\beta$ in EpithelialMesenchymal Transition (EMT): Down-regulation of E-cadherin via snail. Neoplasma, 2015; 62: 1-15

21. An H, Stoops SL, Deane NG et al: Small molecule/ML327 mediated transcriptional de-repression of E-cadherin and inhibition of epithelial-to-mesenchymal transition. Oncotarget, 2015; 6: 22934-48

22. Luo W, Fang W, Li S, Yao K: Aberrant expression of nuclear vimentin and related epithelial-mesenchymal transition markers in nasopharyngeal carcinoma. Int J Cancer, 2012; 131: 1863-73

23. White LR, Blanchette JB, Ren L et al: The characterization of alpha5-integrin expression on tubular epithelium during renal injury. Am J Physiol Renal Physiol, 2007; 292: F567-76
24. Kim MG, Kim SJ, Bae HJ et al: MicroRNA-495-3p functions as tumor suppressor by targeting multiple oncogenic epigenetic effector molecules in gastric cancer. Cancer Res, 2013; 73: 5290

25. Park SM, Shell S, Radjabi AR et al: Let-7 prevents early cancer progression by suppressing expression of the embryonic gene HMGA2. Cell Cycle, 2007; 6: 2585-90

26. Wei JJ, Wu J, Luan C et al: HMGA2: a potential biomarker complement to P53 for detection of early-stage high-grade papillary serous carcinoma in fallopian tubes. Am J Surg Pathol, 2010; 34: 18-26

27. Wu J, Liu Z, Shao C et al: HMGA2 overexpression-induced ovarian surface epithelial transformation is mediated through regulation of EMT genes. Cancer Res, 2011; 71: 349-59

28. Yang D, Wang G, Zhu S et al: MiR-495 suppresses mesendoderm differentiation of mouse embryonic stem cells via the direct targeting of Dnmt3a. Stem Cell Res, 2014; 12: 550-61

29. Xu Y, Ohms SJ, Li Z et al: Changes in the expression of miR-381 and miR495 are inversely associated with the expression of the MDR1 gene and development of multi-drug resistance. PLoS One, 2013; 8: e82062

30. Wang C, Yun Z, Zhao T et al: MiR-495 is a predictive biomarker that downregulates GFI1 expression in medulloblastoma. Cell Physiol Biochem, 2015 36: $1430-39$

31. Yekta S, Shih IH, Bartel DP: MicroRNA-directed cleavage of HOXB8 mRNA Science, 2004; 304: 594-96

32. Hausser J, Landthaler $M$, Jaskiewicz $L$ et al: Relative contribution of sequence and structure features to the mRNA binding of Argonaute/EIF2C miRNA complexes and the degradation of miRNA targets. Genome Res, 2009; 19: 2009-20

33. Jiang X, Huang $\mathrm{H}$, Li Z et al: MiR-495 is a tumor-suppressor microRNA downregulated in MLL-rearranged leukemia. Proc Natl Acad Sci USA, 2012; 109 19397-402

34. Chu $\mathrm{H}$, Chen $\mathrm{X}$, Wang $\mathrm{H}$ et al: MiR-495 regulates proliferation and migration in NSCLC by targeting MTA3. Tumour Biol, 2014; 35: 3487-94

35. Formosa A, Markert EK, Lena AM et al: MicroRNAs, miR-154, miR-299-5p miR-376a, miR-376c, miR-377, miR-381, miR-487b, miR-485-3p, miR-495 and miR-654-3p, mapped to the $14 q 32.31$ locus, regulate proliferation, apoptosis, migration and invasion in metastatic prostate cancer cells. Oncogene 2014; 33: 5173-82

36. Prevot PP, Augereau C, Simion A et al: Let-7b and miR-495 stimulate differentiation and prevent metaplasia of pancreatic acinar cells by repressing HNF6. Gastroenterology, 2013; 145: 668-78e663

37. Hwang-Verslues WW, Chang PH, Wei PC et al: miR-495 is upregulated by E12/E47 in breast cancer stem cells, and promotes oncogenesis and hypoxia resistance via downregulation of E-cadherin and REDD1. Oncogene, 2011; 30: 2463-74 